\title{
The Sigma-1 Receptor Antagonist, S1RA, Reduces Stroke Damage, Ameliorates Post-Stroke Neurological Deficits and Suppresses the Overexpression of MMP-9
}

\author{
Pilar Sánchez-Blázquez ${ }^{1,2}$ (D) Andrea Pozo-Rodrigálvarez ${ }^{1} \cdot$ Manuel Merlos $^{2}$ • \\ Javier Garzón ${ }^{1}$
}

Received: 16 January 2017 / Accepted: 26 July 2017 / Published online: 5 August 2017

(C) The Author(s) 2017. This article is an open access publication

\begin{abstract}
The glutamate N-methyl-D-aspartate receptor (NMDAR) plays an essential role in the excitotoxic neural damage that follows ischaemic stroke. Because the sigma-1 receptor $(\sigma 1 \mathrm{R})$ can regulate NMDAR transmission, exogenous and putative endogenous regulators of $\sigma 1 \mathrm{R}$ have been investigated using animal models of ischaemic stroke. As both agonists and antagonists provide some neural protection, the selective involvement of $\sigma 1 \mathrm{Rs}$ in these effects has been questioned. The availability of S1RA (E-52862/MR309), a highly selective $\sigma 1 \mathrm{R}$ antagonist, prompted us to explore its therapeutic potential in an animal model of focal cerebral ischaemia. Mice were subjected to right middle cerebral artery occlusion (MCAO), and post-ischaemic infarct volume and neurological deficits were determined across a range of intervals after the stroke-inducing surgery. Intracerebroventricular or intravenous treatment with S1RA significantly reduced the cerebral infarct size and neurological deficits caused by permanent MCAO (pMCAO). Compared with the control/shamoperated mice, the neuroprotective effects of S1RA were observed when delivered up to $5 \mathrm{~h}$ prior to surgery and $3 \mathrm{~h}$ after ischaemic onset. Interestingly, neither mice with the genetic
\end{abstract}

Pilar Sánchez-Blázquez and Andrea Pozo-Rodrigálvarez contributed equally to this study

Pilar Sánchez-Blázquez

psb@cajal.csic.es

1 Neuropharmacology. Instituto Cajal, Consejo Superior de Investigaciones Científicas (CSIC), Doctor Arce, 37, 28002 Madrid, Spain

2 Drug Discovery and Preclinical Development, Esteve, Scientific Park of Barcelona, Baldiri i Reixac 4-8, 08028 Barcelona, Spain deletion of $\sigma 1 \mathrm{R}$ nor wild-type mice that were pre-treated with the $\sigma 1 R$ agonist PRE084 showed beneficial effects after S1RA administration with regard to stroke infarction. S1RAtreated mice showed faster behavioural recovery from stroke; this finding complements the significant decreases in matrix metalloproteinase-9 (MMP-9) expression and reactive astrogliosis surrounding the infarcted cortex. Our data indicate that S1RA, via $\sigma 1 \mathrm{R}$, holds promising potential for clinical application as a therapeutic agent for ischaemic stroke.

Keywords Sigma 1 receptor · S1RA · Stroke · Neuroprotective effects · MMP-9 $\cdot$ Astrogliosis

\section{Introduction}

Stroke is one of the leading causes of death worldwide with ischaemic stroke accounting for the clear majority of cases [1, 2]. The global 30-day mortality rate for stroke is estimated at around $23 \%$, but mortality rates can vary according to age, gender and country [3]. Although a variety of rehabilitation strategies exist, no currently approved drug-based therapy can stimulate the recovery of neurological functions in patients following stroke [4, 5]. Local inflammatory changes and neural circuit plasticity that occur post-stroke affect the extent of the brain and behavioural recovery. Experimental data suggest that N-methyl-D-aspartate receptor (NMDAR) stimulation can lead to oxidative stress, which can trigger the upregulation of matrix metalloproteinases (MMPs) early after cerebral ischaemia and reperfusion [6, 7]. MMPs, in particular MMP-9, are important mediators of microvascular blood-brain barrier (BBB) injury and haemorrhagic transformation after ischaemic stroke $[8,9]$. In addition, the neurotoxicity of glutamate 
and other excitatory amino acids might play an important role in the pathogenesis of ischaemic neuronal injury [10-12]. Therefore, drugs that antagonise glutamate NMDAR transmission (e.g. memantine) may reduce the volume of cerebral infarction that results from experimental stroke [13].

Evidence suggests that sigma-1 receptors ( $\sigma 1 \mathrm{Rs})$ are involved in several neurological and psychiatric conditions [14]. Moreover, they have the potential to modulate NMDAR transmission [15]. Recent work has revealed that the histidine triad nucleotide-binding protein 1 (HINT1)- $\sigma 1 \mathrm{R}$ complex mediates the enhancement that certain $G$ protein-coupled receptors (GPCRs) promote on glutamate NMDAR function [16]. In this context, $\sigma 1 \mathrm{R}$ antagonism prevents the HINT1 protein from enabling GPCRs to enhance the function of NMDARs, thereby preventing the further enhancement of this glutamatergic activity [16]. In fact, in neuropathic and inflammatory pain models involving NMDAR activation, antagonists of the $\sigma 1 \mathrm{R}$ are effective in reducing pain behaviours $[17,18]$. More relevantly, $\sigma 1 \mathrm{R}$ ligands have been shown to enhance neuroplasticity and functional recovery following experimental stroke [19]; however, at the doses used, the agonists and antagonists of $\sigma 1 \mathrm{Rs}$ produce comparable effects. Moreover, the selectivity of some of these pharmacological tools is far from optimal [17]. As a result, the involvement of $\sigma 1 \mathrm{Rs}$ in these positive effects is debated.

Based on these findings, we explored the possibility that the selective $\sigma 1 \mathrm{R}$ antagonist, S1RA (E-52862/ MR309) [20], could shed light on the role that this receptor plays in the inflammatory response after experimental stroke. We used an animal model of focal ischaemia induced via middle cerebral artery occlusion (MCAO) to reproduce the most frequent form of stroke in humans $[21,22]$. Highly reproducible cortical lesions were obtained by directly ligating the middle cerebral artery (MCA) after temporal craniotomy. This primarily damaged the frontal and parietal cortices, thereby resulting in reliable infarct size across animals but limiting their associated sensory deficits [22]. The aim of this study was to determine whether the central and systemic administration of S1RA had therapeutic effects on cerebral infarct and oedema volumes as well as the ability to ameliorate their associated neurological deficits.

\section{Materials and Methods}

\section{Animals}

Wild-type male CD1 mice and knockout mice with the genetic background of CD1 mice and exhibiting targeted disruption of the $\sigma l R$ gene were used in this study (Envigo, Barcelona, Spain). Mice were housed at a constant temperature $\left(22 \pm 1{ }^{\circ} \mathrm{C}\right)$ under a $12 / 12$-h light-dark cycle and were allowed unlimited access to food and water. Animal experiments were performed in accordance with the procedures for the Care and Use of Laboratory Animals of the European Commission guidelines (Directive 2010/63/EU). The Committee on Animal Care at Consejo Superior de Investigaciones Científicas (CSIC) approved all procedures for handling and sacrificing the animals.

\section{Permanent MCAO and the Determination of Infarct Size}

Focal cerebral ischaemia was induced via MCAO, as described previously [23]. Briefly, mice were anesthetised, and a vertical skin incision $(0.5 \mathrm{~cm})$ was made between the right eye and ear under a dissection microscope. A small craniotomy was performed over the trunk of the right MCA and above the rhinal fissure. The artery was ligated just before its bifurcation between the frontal and parietal branches with a 9-0 suture. Sham-operated animals were subjected to an identical procedure, except that the MCA was not ligated. The mice were returned to their cages after surgery, kept at room temperature and allowed food and water ad libitum. To determine the infarct size $48 \mathrm{~h}$ after MCAO, magnetic resonance imaging (MRI) was performed using a BIOSPEC BMT 47/40 (Bruker, Ettlingen, Germany). We used the dorsal third ventricle as an internal anatomical marker to align, register and compare the collection of images from each mouse. The infarct volume was calculated using ImageJ 1.4 as the percentage of the hemisphere that is infarcted based on the T2weighted images.

Two days after permanent MCAO (pMCAO), one set of animals were euthanised prior to their brains being removed and seven 1-mm-thick coronal brain slides (Brain Matrix, WPI, UK) were obtained. The sections were stained with $1 \%$ 2,3,5-triphenyltetrazolium chloride (TTC; Sigma, Spain). Infarct volumes were calculated by sampling each side of the coronal sections with a digital camera (Nikon Coolpix 990, Tokyo, Japan). The extent of unstained infarct area (expressed in $\mathrm{mm}^{2}$ ) was integrated from the total area as an orthogonal projection.

\section{Drugs}

The newly synthesised $\sigma 1 \mathrm{R}$ antagonist, S1RA: 4-[2-[[5-methyl-1-(2-naphthalenyl)-1H-pyrazol-3-yl]oxy] ethyl] morpholine), was obtained from Laboratorios Esteve (Barcelona, Spain). BD1047 (\#0956), BD1063 (\#0883) and PRE084 (\#0589) were obtained from Tocris Bioscience (Bristol, UK). Compounds were dissolved in ethanol/Cremophor EL/physiological saline (1:1:18). To facilitate selective and straightforward access to their targets, the compounds were each injected into the lateral ventricles of mice at $4 \mu \mathrm{L}$ as previously described or via an injection in the tail vein. Groups of 8 to 10 mice received doses of the selected compounds. 


\section{Behavioural Outcomes}

Behavioural tests were conducted during the first week after pMCAO in S1RA-treated ( $3 \mathrm{nmol} / \mathrm{m} \mathrm{icv,} 1 \mathrm{~h}$ post-surgery) and untreated mice and outcomes compared with shamoperated mice. The primary screening included body weight and contact-righting reflex measurements. Body temperature of each mouse was measured right before, 1, 3 and $5 \mathrm{~h}$ following injection using a digital readout thermocouple (BAT12 thermometer, Physitemp Instruments, Clifton, NJ, USA) with a resolution of $0.1^{\circ} \mathrm{C}$ and accuracy of $\pm 0.1{ }^{\circ} \mathrm{C}$ attached to a RET-3 Rodent Sensor. Any incidence of abnormal behaviour, fear, irritability, aggression or vocalisation drives to the exclusion of this particular mouse.

Activity Meter Mice were tested individually; locomotion scores were measured using a multicage activity meter (Accuscan activity analyser-Versamax260 v2.4; Omnitech Electronics, Inc., USA). Beam breaks were recorded for $30 \mathrm{~m}$ as a measure of basal locomotor activity. Separate counterclockwise $(\mathrm{CCW})$ and clockwise $(\mathrm{CW})$ rotations were also determined. The first measures were obtained 1 day before surgery and 3 days after surgery (i.e. days 2, 3 and 5). The chamber was cleaned with diluted alcohol $(70 \% v / v)$ and dried between trials.

Hot Plate Test Animals were individually placed on a hot plate with the temperature adjusted to $53 \pm 0.5{ }^{\circ} \mathrm{C}$. A transparent plastic cylinder (14-cm diameter, 20-cm height) was used to confine a mouse on the heated surface of the plate. Heat exposure continued until the nocifensive reaction (licking) of either hind paw occurred.

Acoustic Startle Response The startle device consisted of a non-restrictive Plexiglas cage that enclosed the sensor's platform but did not touch it. The device was located in a soundattenuating chamber constantly illuminated via a 10-W lamp. The chamber was also equipped with a loudspeaker that constantly provided $46 \mathrm{~dB}[\mathrm{~A}]$ of background white noise. Two 28 $\mathrm{cm}$ loudspeakers (Cibertec, Madrid, Spain), located $15 \mathrm{~cm}$ from both sides of the Plexiglas cage, produced the various acoustic stimuli. These speakers were connected to an audio amplifier (Cibertec), which was connected to a noise generator (2001 Function Generator, Columbus, OH, USA). Mice were tested for their acoustic startle response 5 days after surgery. After an initial 5-m period of accommodation to the 46-dB background noise, 15 startle/unpredictable eliciting stimuli were presented (typically $50-\mathrm{ms}$ bursts of 73-120-dB white noise).

Passive Avoidance Task Acquisition and retention of passive avoidance behaviours were examined 5 days after surgery, using two-compartment box with a partition which embodies a sliding door (Ugo Basile, Rome Italy). In the acquisition trial, each mouse was initially placed in the light compartment, and the door between the two compartments was opened after $10 \mathrm{~s}$. When the mouse entered the dark compartment, the guillotine door automatically closed and an electrical foot shock $(0.5 \mathrm{~mA}, 1 \mathrm{~s})$ was delivered through the floor. The latency time before crossing into the dark chamber was recorded. For the retention trial, each mouse was again placed in the light compartment, and the latency time before crossing into the dark compartment was recorded (up to $5 \mathrm{~m}$ ).

Rotarod Motor coordination was measured using an accelerated rotarod (Ugo Basile). Before surgery, each animal was trained to use the rotarod via six 5-m sessions at a constant acceleration and with an interval of $20 \mathrm{~m}$ between trials. On the following days, the mice were again tested and the time to fall from the rod was measured with a cutoff time established at $5 \mathrm{~m}$.

Pole Test The animals were placed head-up on top of a vertical rough-surfaced pole (diameter, $8 \mathrm{~mm}$; height, $55 \mathrm{~cm}$ ), and the time to orient downward ( $t$-turn) and total time to descend were measured. The fastest performance over the three trials was used; mice that dropped from the pole were considered to take $120 \mathrm{~s}$ (default value), the highest value.

\section{Tissue Fixation and Immunohistochemistry}

Mice ( $n=6$ per group) were euthanised at $48 \mathrm{~h}$ and 5 or 8 days after the induction of pMCAO. Brains were removed after transcardial perfusion, post-fixed by immersion for $3 \mathrm{~h}$ at room temperature and cryopreserved in $30 \%$ sucrose in $0.1 \mathrm{M}$ phosphate buffer $\mathrm{pH}$ 7.4. Brains were frozen and cut in a cryostat (CM1950; Leica Biosystems) and 40- $\mu$ m coronal sections were collected. Sections were air-dried and blocked at $4{ }^{\circ} \mathrm{C}$ overnight using a solution of $0.25 \%$ Triton X-100 and $5 \%$ donkey serum in Tris-buffered saline (TBS) followed by incubation with anti-glial fibrillary acidic protein (GFAP; Dako Z0334), anti-collagen IV (Abcam AB6586) and antineuronal nitric oxide synthase (nNOS; Thermo 61-7000) for $24 \mathrm{~h}$ at $4{ }^{\circ} \mathrm{C}$. The signal was revealed using the avidin-biotin peroxidase complex (ABC) technique. A 1:150 dilution of biotinylated Lycopersicon esculentum (tomato) lectin (Sigma-Aldrich L0651) was used to stain some sections to study vessel distribution, and processed with an ABC kit followed by diaminobenzidine-nickel. The pictures were taken using a digital camera (Polaroid DMC IE, Cambridge, MA, USA) coupled to a microscope (Axioplan 2, Zeiss, Jena, Germany).

\section{Synaptosome Preparation and Western Blots}

Membranes from the frontal cortex were prepared as previously described [16], and the separated proteins were transferred onto $0.2-\mu \mathrm{m}$ polyvinylidene difluoride membranes (Bio-Rad \#162-0176, Madrid, Spain). The membranes were 
probed for $24 \mathrm{~h}$ at $6{ }^{\circ} \mathrm{C}$ with the selected antibodies diluted in TBS $+0.05 \%$ Tween 20 (TTBS) in DecaProbe chambers (PR 150, Hoefer-GE, Barcelona, Spain). The primary antibodies were as follows: anti-MMP-9 (1:1000, Millipore AB13458), PhosphoDetect anti-p38 MAP kinase which specifically recognises the dual-phosphorylated (on residues Thr180 and Tyr182) active form of the enzyme (1:1000, Millipore 506119), Phospho-CaMKII (Thr286) antibody (1:1000, Cell Signalling \#12716), anti-nNOS (1:1000) and anti-GFAP (1:1000). Signals were detected using horseradish peroxidase
(HRP)-conjugated secondary antibodies (1:10,000 in TTBS). Antibody binding was visualised using Immobilon Western Chemiluminescent HRP substrate (Millipore WBKLS0100), and chemiluminescence recorded using a ChemiImager IS5500 (Alpha Innotech, CA, USA) equipped with a Peltiercooled CCD camera that provided a real-time readout of 30 frames per second $\left(-35^{\circ} \mathrm{C}\right.$; high signal-to-noise ratio; dynamic range of up to 3.4 optical density units). Densitometry (average optical density of the pixels within the object area $/ \mathrm{mm}^{2}$ ) was performed using Quantity One (Bio-Rad, Madrid, Spain).

a

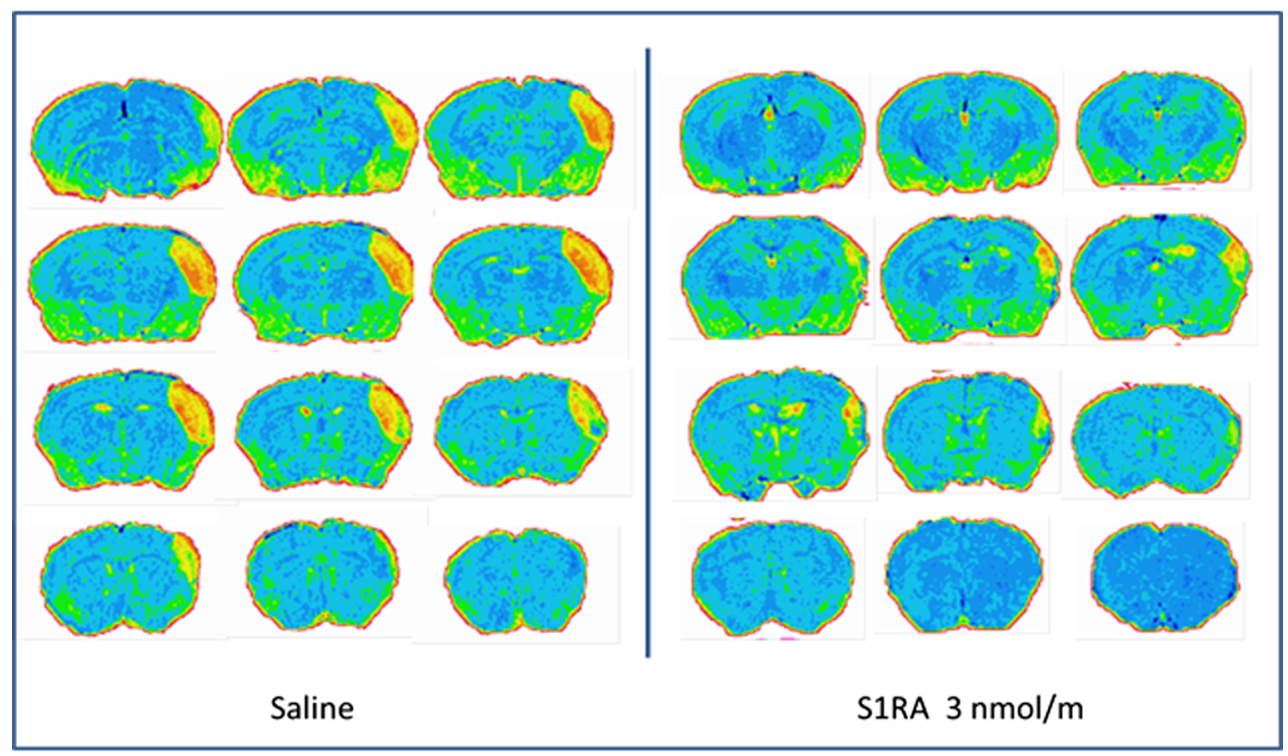

b

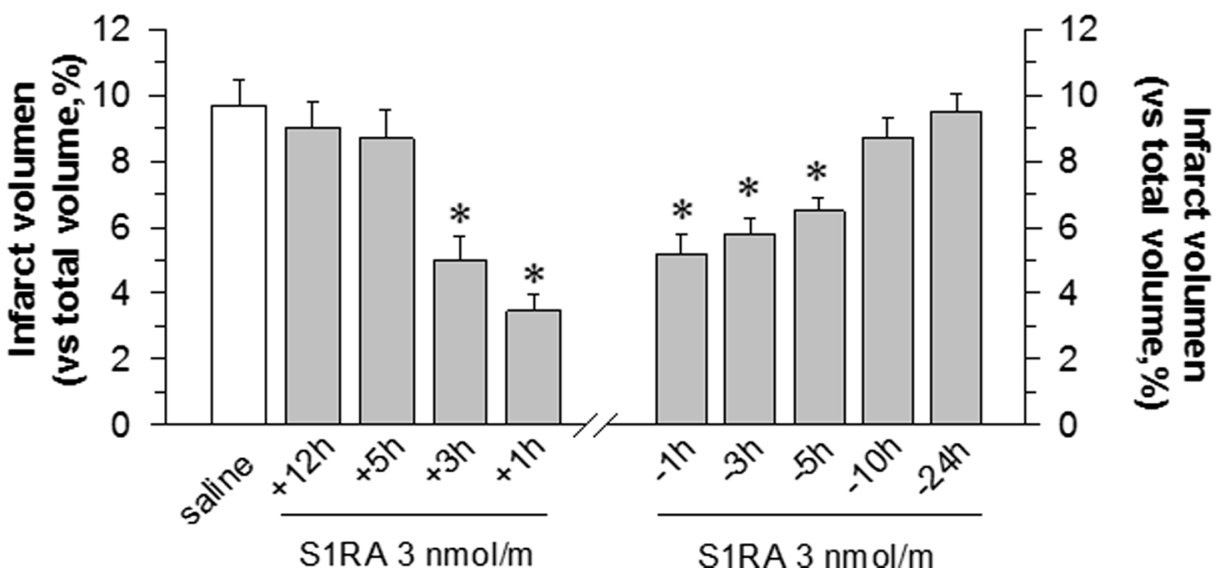

Fig. 1 The administration of S1RA diminishes ischaemic brain damage. a Representative brain section images were obtained from saline- (left) and S1RA-treated mice $(3 \mathrm{nmol} /$ mouse, $1 \mathrm{~h}$ after surgery; right) $48 \mathrm{~h}$ after pMCAO using MRI (BIOSPEC BMT 47/ 40). b The bar graphs quantitatively compare the infarct volume $( \pm \mathrm{SEM})$ from the saline- (white bars) and S1RA-treated mice (grey

bars) at different time intervals before and after surgery. Groups consisted of 8-10 mice, and the data are represented as the means \pm SEMs. Asterisk indicates the significant difference from saline-treated mice, degrees of freedom $(d f)=16$, all pairwise Holm-Sidak multiple comparison tests following ANOVA, $p<0.05$ 


\section{Statistical Analyses}

All graphs and statistical analyses were generated and performed using the SigmaPlot/SigmaStat v.13 package (SPSS Science Software, Erkrath, Germany). Significance was defined as $p<0.05$. Data were analysed using paired $t$ test or one-way ANOVA followed by all pairwise Holm-Sidak multiple comparison tests.

\section{Results}

\section{Determination of Infarct Volume}

The volumetric analysis of the in vivo MRI data showed that neither surgery nor intracerebroventricular (icv) administration significantly changed the total brain volume $(437.9 \pm 31.1$ and. $456.7 \pm 24.8$, respectively; sham-operated mice $441.4 \pm 26.3 \mathrm{~mm}^{3}$ ). However, pMCAO produced severe injury in mice when examined $48 \mathrm{~h}$ after ischaemia (Fig. 1a). Injury was mostly apparent in the cerebral cortex, and the infarct volume was estimated as affecting $9.7 \pm 1.8 \%$ of the total brain volume. No damage was observed in the sham-operated mice. Compared with untreated mice, the administration of S1RA $1 \mathrm{~h}$ post-surgery improved stroke outcomes (an approximate $50 \%$ reduction in the infarct size to $3.48 \pm 0.9 \%$ of the total brain volume) after permanent cerebral ischaemia (Fig. 1a).

To examine the time course of the neuroprotective effect of S1RA, the compound was icv injected at doses of 1, 3 and 10 nmol per mouse. Whereas 1 and 3 nmol showed beneficial effects, the dose of $10 \mathrm{nmol}$ was less effective. Thus, a dose of 3 nmol S1RA was selected for the study, and this dose coincided with that used in animal models of neuropathy and opioid analgesia regulation $[16,24]$. Although the protective effect was observed when S1RA was administered up to $5 \mathrm{~h}$ before surgery, the beneficial effects disappeared when administered more than $3 \mathrm{~h}$ after the initiation of the ischaemic procedure (Fig. 1b).

Next, we addressed whether the systemic application of S1RA protected against neuronal damage at the end of pMCAO because this protocol has more potential for the clinical treatment of patients recovering from stroke. Intravenous (iv) administration of S1RA $1 \mathrm{~h}$ after pMCAO protected against ischaemic injury, showing a similar efficacy as the icv route (Fig. 2a). Compared with untreated mice, S1RA (30 $\mathrm{\mu g}$ per mouse, iv) were clearly protected against stroke outcomes; the infarct sizes were $6.71 \pm 1.7$ and $3.37 \pm 0.9 \%$ of the total brain volume, respectively.
Fig. 2 A Left: TTC stained brain section images were obtained from saline and S1RA treated mice ( $30 \mathrm{mg} /$ mouse, iv; $1 \mathrm{~h}$ after surgery) $48 \mathrm{~h}$ after pMCAO. White indicates infarction; red staining indicates normal tissue. Right: Quantification of infarct volumes based on TTC staining. $\mathrm{B}$ Compounds were injected at a dose of $3 \mathrm{nmol} /$ mouse $1 \mathrm{~h}$ after surgery. The bars quantitatively compare the infarct volume in WT mice. $\sigma 1 \mathrm{R}^{-/-}$animals showed significantly greater infarct areas that were not reduced by S1RA administration. Groups consisted of 8-10 mice, and the data are represented as the means \pm SEMs. Asterisk indicates the significant difference from saline-treated mice, $\theta$ significantly different from WT mice; degrees of freedom $(d f)=16$, all pairwise Holm-Sidak multiple comparison tests following ANOVA, $p<0.05$ a

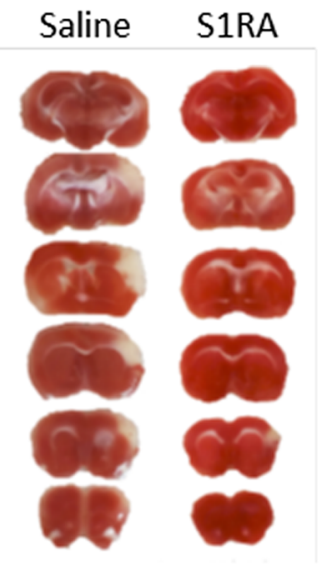

b

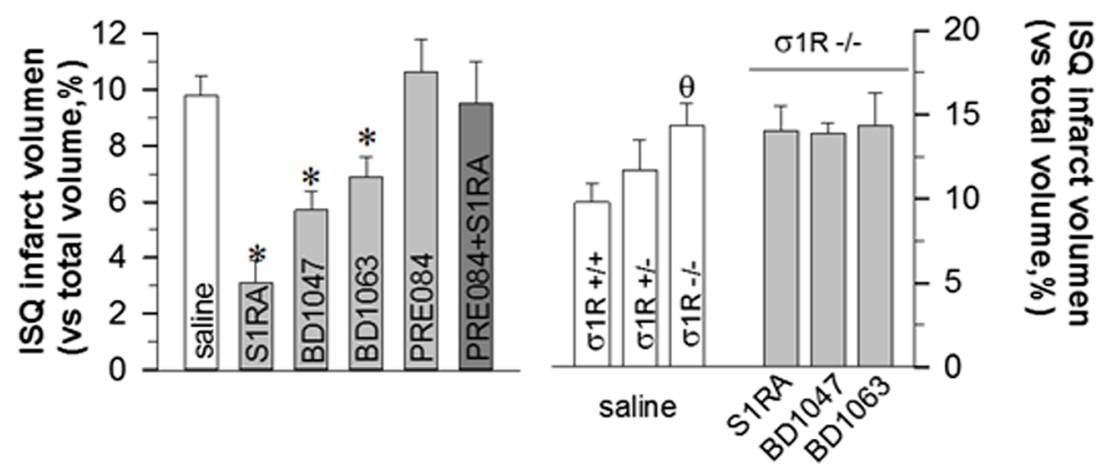


We previously reported that S1RA is likely the most potent and selective $\sigma 1 \mathrm{R}$ antagonist available [24] as other antagonists (e.g. BD1047 and BD1063) that have been used at $3 \mathrm{nmol}$ per mouse produced weaker protective effects. Based on the experimental conditions and doses we used, the PRE084 agonist showed no direct effect on infarct volume; however, it did prevent S1RA neuroprotection (Fig. 2b). The involvement of the $\sigma 1 R s$ in the effects of S1RA was ascertained using $\sigma 1 \mathrm{R}^{-/-}$mice. These mice showed increased infarct volumes with respect to their wild-type (WT) controls, which were refractory to the $\sigma 1 \mathrm{R}$ ligands included in this study (Fig. 2b).

\section{Behavioural Assessment}

In addition to reducing infarct size, we also sought to determine whether S1RA protects or improves the performance of mice in various sensory-motor tests. No differences were observed between the stroke and sham-operated animals with regard to muscle strength or movement latency. Neither alteration in the body temperature of subjects injected with S1RA was observed when compared to saline-injected controls. Rectal temperature was measure $30 \mathrm{~min}$ after surgery and then hourly up to $7 \mathrm{~h}$. However, a significant effect of pMCAO was found with regard to body weight (Fig. 3a). Data analyses revealed that the stroke animals weighed significantly less than the sham-operated animals on post-surgical days 2 through 5 . To discard the influence of body weight in the behavioural performance, a parallel group of mice with limited access to food were used. Our data indicate that body weight difference does not affect the global behavioural outcomes. Subsequently, we evaluate the influence of pMCAO to heat and acoustic stimuli. In operated animals, an increased sensitivity to both stimulus was observed (Fig. 3b, c). The administration of S1RA ( $3 \mathrm{nmol} /$ mouse, icv, $1 \mathrm{~h}$ after surgery) attenuated all these altered behaviours. No significant difference was observed between the pMCAO and the sham groups with regard to the learning performance task. The latency to enter the dark, electrified chamber was significantly longer on the test day than the training day (5 days post-stroke). However, the majority of the animals entered the dark chamber before the end of the 5-min period, suggesting that retention of this learning task is similar regardless of ischaemic damage (Fig. 3d). According to the verticalpole task, pMCAO significantly increased the time needed to climb down to the base of the pole (Fig. 4a). The mice developed a hyperactive phenotype, as has been previously reported [25], with a rotation preference and apparent motor deficits (Fig. 4b-d), and S1RA administration significantly attenuated all these behaviours.
Fig. 3 Behavioural recovery from stroke via S1RA treatment. The response rate functions of the three groups of mice regarding a body weight gain in grams $(\mathrm{g}), \mathbf{b}$ hot plate latency in seconds (s), c startle response at 73 and $120 \mathrm{~dB}$ and expressed as arbitrary units (au) and $\mathbf{d}$ learning performance in the passive avoidance test. Groups of 8-10 mice were used for each treatment, and the data are represented as the means \pm SEM. Asterisk indicates the significant difference from the sham-operated mice, degrees of freedom $(d f)=16$, all pairwise Holm-Sidak multiple comparison tests following ANOVA, $p<0.05$
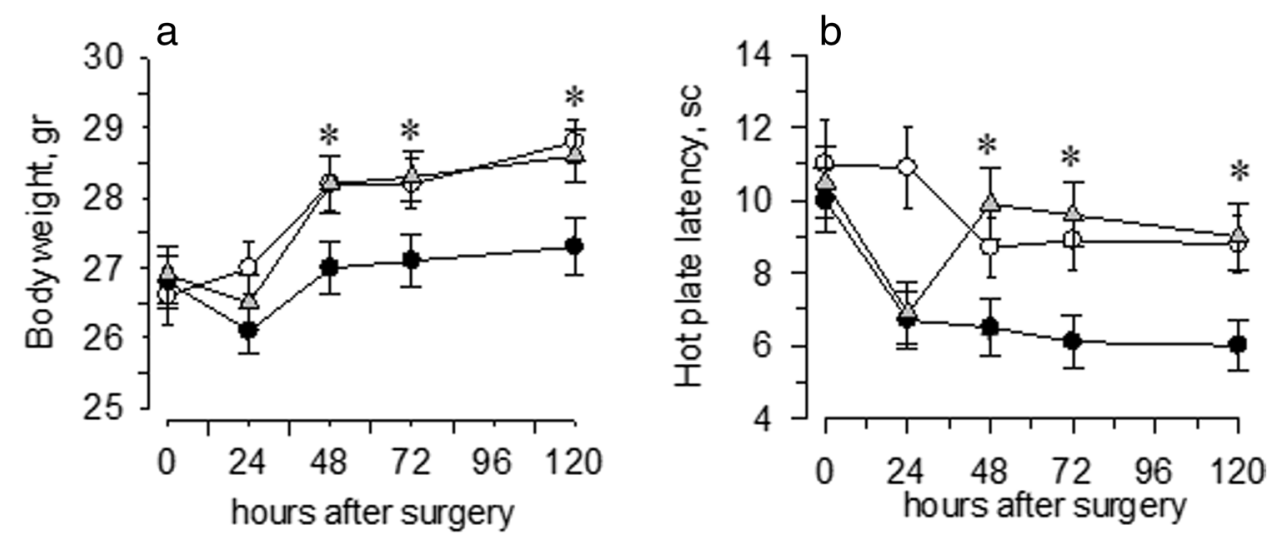
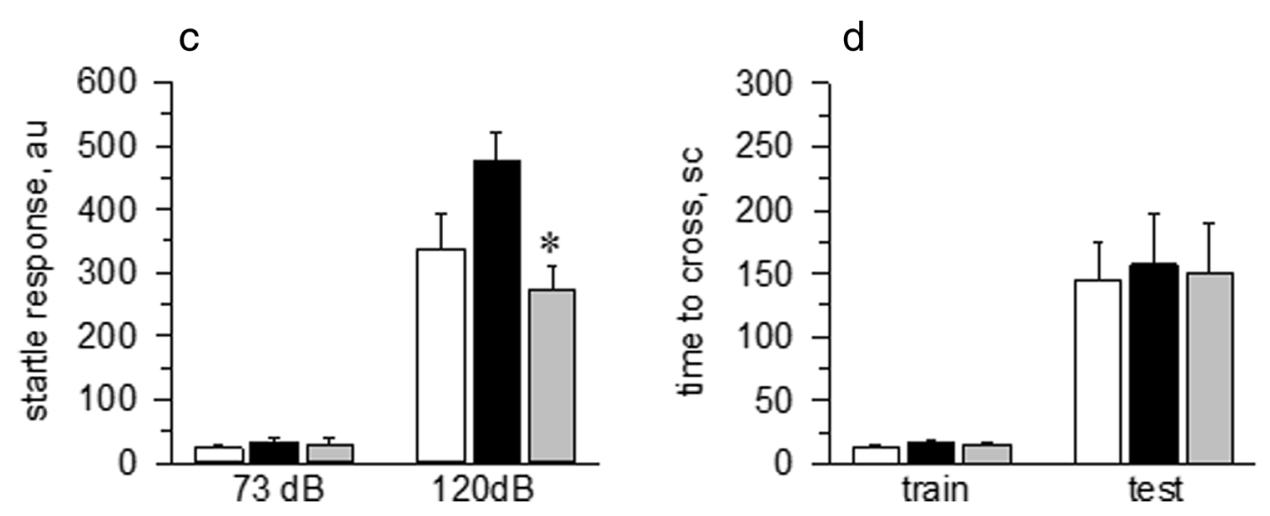
Fig. 4 Improved recovery of sensory-motor function from stroke after S1RA treatment. The response rate functions of the three groups of mice regarding the a seconds (s) to descent to the floor in the pole test, $\mathbf{b}$ locomotor activity, c counterclockwise $(\mathrm{CCW})$ rotation preference and $\mathbf{d}$ latency in seconds (s) to fall in rotarod. Groups of 8-10 mice were used for each treatment, and the data are represented as the means \pm SEM. Asterisk denotes the significant difference from the sham-operated mice, degrees of freedom $(d f)=16$, all pairwise Holm-Sidak multiple comparison tests following ANOVA, $p<0.05$
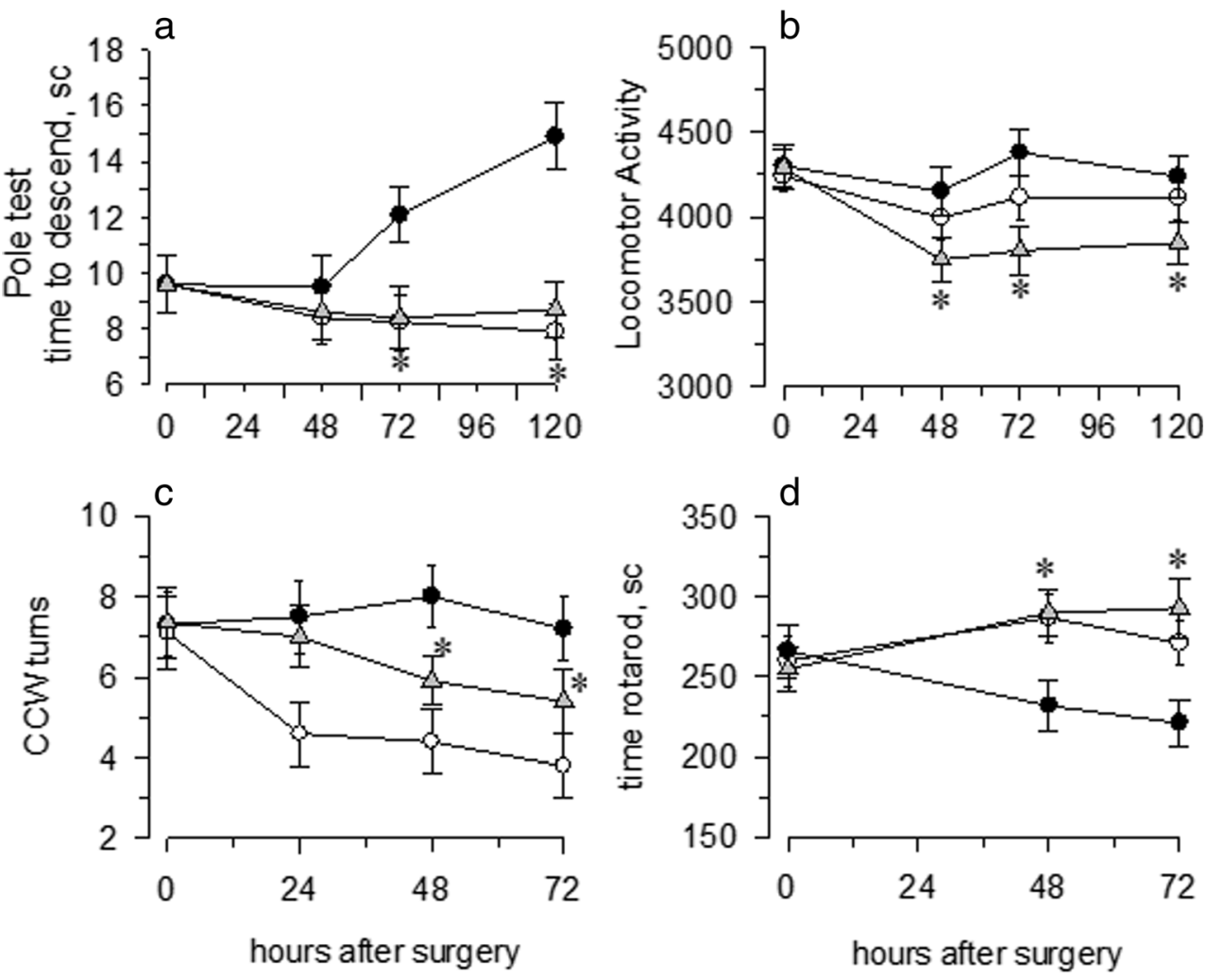

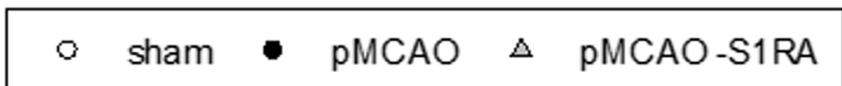

The Effects of S1RA on Post-Ischaemic BBB Disruption, the Inflammatory Response and Astrogliosis

Metalloproteinases are associated with cerebrovascular disruption and neuronal damage, and thus, the effects of pMCAO on the MMP-9 protein were determined in western blots. We found that $\mathrm{pMCAO}$ produced a significant increase in the activated $86 \mathrm{kDa}$ MMP-9 in the frontal cortex without altering the inactive 92-kDa form (Fig. 5a). These changes were evident $48 \mathrm{~h}$ after surgery and they persisted for up to 9 days after stroke. However, the administration of S1RA ( $3 \mathrm{nmol} / \mathrm{m}$, icv) $1 \mathrm{~h}$ after surgery virtually abolished the overexpression of active MMP-9. We also examined p38 MAPK activation as a marker of inflammation, nNOS expression and the endogenous levels of Thr286 phosphorylated CaMKII, indicative of NMDAR over-activation. In addition, glial inflammatory changes were also evaluated in the brain following ischaemic insult, as was the influence of S1RA administration on the reactive astrogliosis induced by pMCAO. Finally, microglial activation was determined through the tomato lectin staining of vessels and microglia, and as expected, pMCAO induced strong reactive astrogliosis as witnessed by GFAP upregulation (Fig. 5b). Immunohistochemical staining of the mouse cortex $48 \mathrm{~h}$ after pMCAO showed increased immunoreactivity in astrocytes (GFAP-positive cells) and activated microglia
(L. esculentum lectin-positive cells; Fig. 6). Significantly, treatment with S1RA diminished the GFAP expression and dampened the activated microglia, as well as the immunoreactivity for nNOS, phosphorylated p38 and pCaMKII (Figs. 5 and 6).

\section{Discussion}

In this study, we used a permanent model of ischaemia to simulate the most common type of localised stroke in humans, specifically the permanent occlusion of the cerebral arteries $[26,27]$. We found that the administration of S1RA, a selective antagonist of $\sigma 1 \mathrm{R}$, markedly reduced the infarct volume and improved functional recovery. Vehicle-treated animals showed significant post-stroke impairments in motor and sensitive function status, whereas S1RA, given either $1 \mathrm{~h}$ after the onset of pMCAO, reduced these deficiencies.

Compounds that are selective agonists or antagonists of $\sigma 1 \mathrm{R}$ have been used to ascertain whether it is the activation or inhibition of this receptor that results in protection against oxidative stress. Overall, the available in vitro data indicate that inhibition, not activation, of $\sigma 1 \mathrm{Rs}$ prevents oxidative stress-induced cell death [28]. In that sense, neurosteroids are well known to bind to $\sigma 1$ Rs in addition to hormonal receptors [29]. The neurosteroid progesterone has been 
a

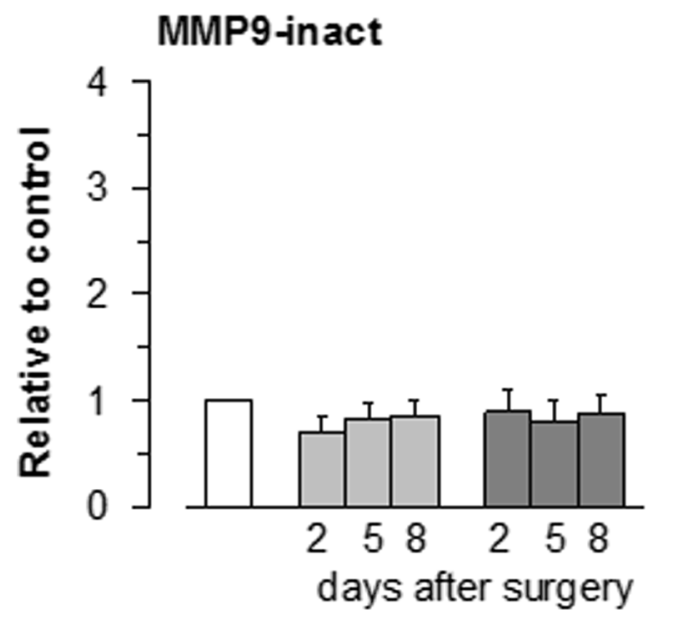

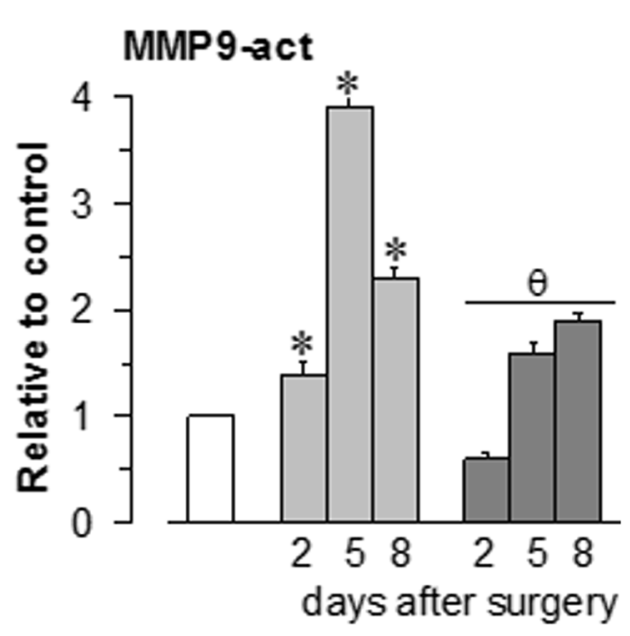

b

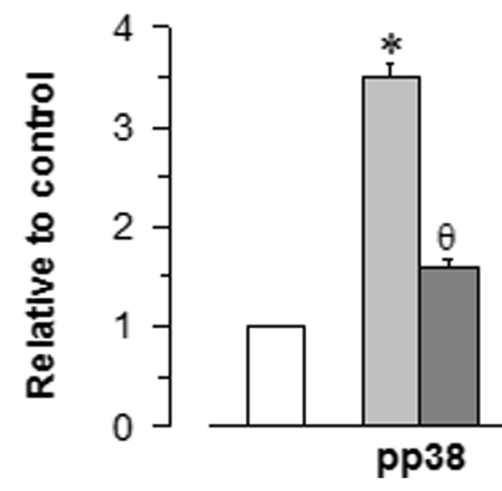

Fig. 5 Stimulation of MMP-9 protein expression and inflammatory markers via pMCAO. a The expression levels of both the active $(86 \mathrm{kDa})$ and the inactive $(92 \mathrm{kDa})$ forms of MMP-9 were determined at different times after surgery. $\mathbf{b}$ The ischaemic-induced phosphorylation of p38 and CaMKII as well as the expression levels of nNOS and GFAP were evaluated 8 days after surgery. The enhancing effects of pMCAO (light grey) were reduced by S1RA administration (dark grey). Each bar represents the means \pm SEM of the data from three determinations

considered an antagonist of $\sigma 1$ Rs and has exhibited neuroprotective effects in a number of studies using experimental stroke and CNS trauma models [30-32]. Similarly, the acute administration of the dopamine D2 receptor antagonist haloperidol, at doses that bind to $\sigma 1 \mathrm{R}$ as an antagonist, also displays neuroprotection [28]. Unfortunately, the available data do not allow us to ascribe the neuroprotective effects of these substances to $\sigma 1$ Rs alone. Importantly, various experimental models of acute brain injury or neurodegeneration have also shown the neuroprotective actions of the $\sigma 1 \mathrm{R}$ agonists. Rats subjected to $2 \mathrm{~h}$ of transient MCAO (tMCAO), continuously treated with the agonist $(+)$ pentazocine or 4-phenyl-1-(4phenylbutyl) piperidine (PPBB) for $22 \mathrm{~h}$ starting $1 \mathrm{~h}$ after occlusion $[33,34]$, showed a reduced infarct volume. A reduction in the infarct size was also found in rats subjected to tMCAO and treated with the PRE084 agonist at $3 \mathrm{~h}$ into reperfusion, [35]. Furthermore, dimemorfan has shown protective effects against ischaemic stroke in rats. It has been postulated that these effects are a result of modulation of o1R-dependent signals, which prevent subsequent glutamate accumulation and the downstream pathologic events [36].

It is unclear whether these ligands act as agonists or antagonists or even whether they interact with $\sigma 1 \mathrm{Rs}$ to promote neuroprotection. The protective effects of certain $\sigma 1 \mathrm{R}$ ligands might be because of their effects on other targets such as directly blocking NMDARs. In addition, some data suggest that cutamesine (SA4503), a possible agonist, enhances functional recovery after experimental stroke in rats without affecting infarct size [37]. In a phase 2 clinical trial exploring the safety and functional effects of cutamesine in patients with ischaemic stroke, a trend toward better functional performance 
Fig. 6 Effect of S1RA administration on cerebral markers changes after pMCAO. Photomicrographs of the mouse ipsilateral cortex showing changes in morphology $48 \mathrm{~h}$ after pMCAO. a Lycopersicon esculentum (tomato) lectinlabelled blood vessels and microglial cells (indicated by the arrows). Insert amplified area. $\mathbf{b}$ Glial scar surrounding infarcted zone marked with GFAP. Periinfarct cortical area of brain from the pMCAO + S1RA group showing significant decrease in microglia expression, and a reduction in the glial scar compared with the pMCAO group. Scale bars $100 \mu \mathrm{m}$ (a) and $400 \mu \mathrm{m}$ (b)
Saline S1RA $3 \mathrm{nmol} / \mathrm{m}$
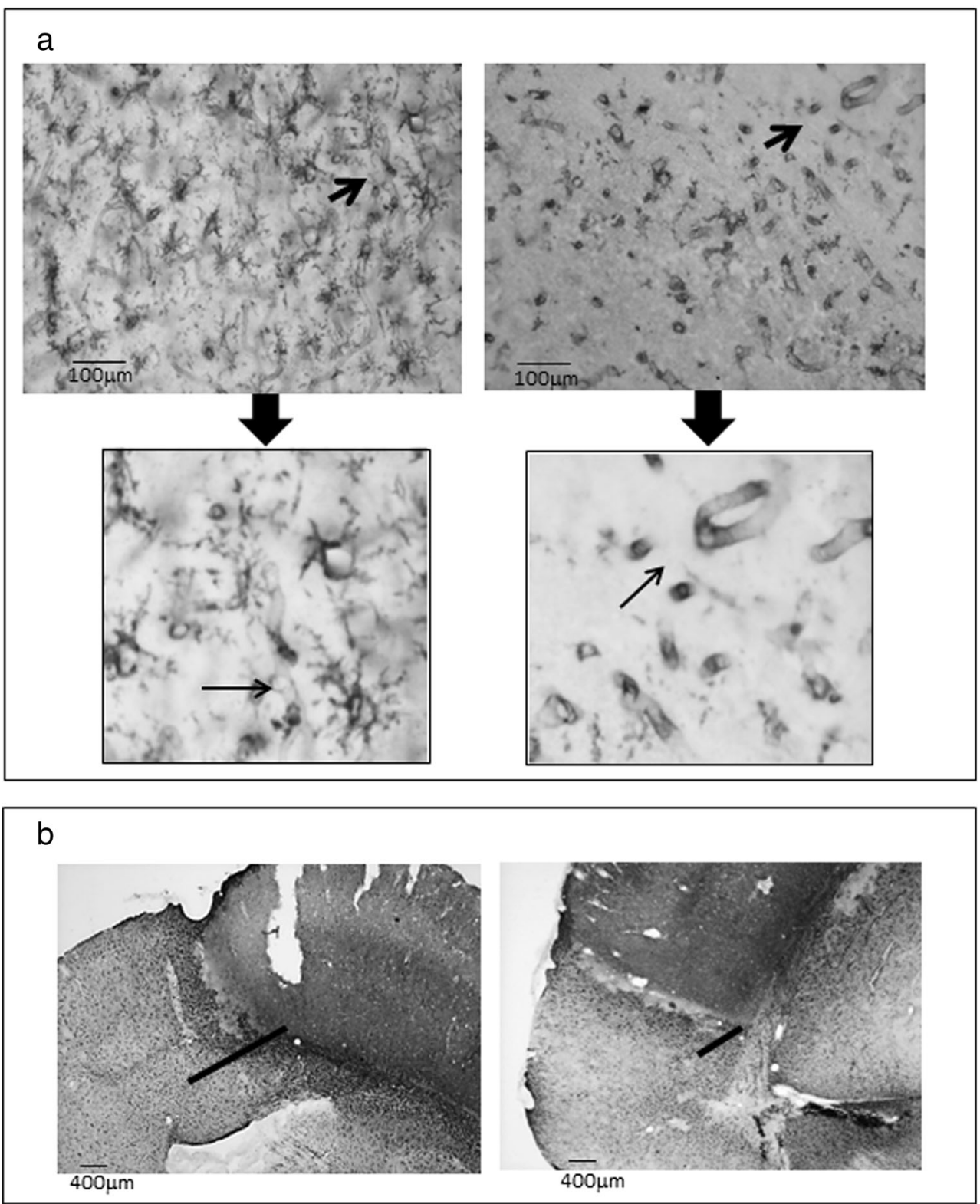

with cutamesine compared with placebo was observed, although there was no significant treatment differences in the primary efficacy measures [38].

In the present work, the compounds assayed were administered at equimolar concentrations via the icv route to discard the differences in bioavailability by crossing the BBB. The effects of S1RA were more potent than those of the other evaluated $\sigma 1 \mathrm{R}$ antagonists. At this dose, PRE084 behaved as an agonist and blocked the positive effects of S1RA and the other $\sigma 1 \mathrm{R}$ antagonists on infarct volume and functional recovery. This finding corroborates previous data showing that S1RA was the most effective antagonist in an in vivo experimental model that discriminated the agonist/antagonist performance of $\sigma 1 \mathrm{R}$ ligands [24].
It is interesting to mention that $\sigma 1 \mathrm{R}^{-/}$mice do not reproduce the beneficial effects observed in wild-type mice treated with S1RA. Probably, this difference is because knockout animals undergo surgery in absence of the $\sigma 1 \mathrm{R}$. Thus, pMCAO might promote increased excitotoxicity in $\sigma 1 \mathrm{R}$-deficient mice via the direct and GPCR/HINT1-mediated activation of NMDAR signalling. In fact, NMDARs show increased responses to NMDA, indicating that $\sigma 1 \mathrm{R}$ dampens the activity of these glutamate receptors (24). Furthermore, the negative influence of cannabinoid type 1 receptors on NMDARs is lost in $\sigma 1 \mathrm{R}^{-/-}$mice $[21,24,39]$. The protective effects of icv administration ( $3 \mathrm{nmol} / \mathrm{mouse}$ ) of the sigma ligands S1RA, BD1047 and BD1063 were absent in mice with genetic $\sigma 1 \mathrm{R}$ deletion. These observations clearly indicate that $\sigma 1 \mathrm{R}$ is 
involved in post-injury ischaemic alterations, and sigma ligands partially modulate the neural injury that the stroke produces.

Following stroke, the disruption of the BBB is a crucial event in the secondary injury cascade, potentiating brain injury through a number of mechanisms, including cerebral oedema, increased neuroinflammatory response and haemorrhagic transformation [40, 41]. MMPs have been implicated in disease states that involve BBB dysregulation, tissue injury and cell death [42]. In particular, the expression and activity of MMP-9 have been found to increase in the ischaemic brain [7]. Accordingly, both inhibitors of MMPs and genetic deletion of MMP-9 attenuate BBB disruption and brain tissue infarction following ischaemia [43, 44]. Furthermore, clinical studies of stroke have found a correlation between MMP-9 levels in the blood and the rate of haemorrhagic transformation [45]. Notably, in this study, S1RA reduced oedema and decreased the MMP-9 activation associated with stroke. Moreover, this $\sigma 1 \mathrm{R}$ antagonist reduced the expression of inflammatory mediators such as nNOS that contribute to postinjury ischaemic conditions. Secondary damage can be caused by inflammation within the brain following cerebrovascular ischaemia and stroke. The precise causes of this inflammatory response have yet to be fully identified, but it is believed that necrotic cells and debris and reactive oxygen species are contributing factors. Within $24 \mathrm{~h}$ of ischaemic injury, these inflammatory triggers can activate microglia, which release an array of inflammatory and cytotoxic mediators that contribute to cell damage and death [46]. Accordingly, activated microglia have been identified in the cerebral cortex of animals exposed to transient focal cerebral ischaemia [47]. Microglial activation induced by cerebral ischaemia is concomitant with astrogliosis (the activation of astrocytes). During this reactive astrogliosis process, astroglial cells increase in size and number and undergo rapid expression of GFAP [47]. The degree of gliosis experienced following brain injury is thought to influence both structural and functional recovery such that certain levels are beneficial to the recovery process but excessive levels may be detrimental due to the associated neuroinflammatory responses [48-50]. Our data indicate that the administration of S1RA reduced reactive astrogliosis after ischaemic injury as demonstrated by decreases in microglial and astroglial proliferation and reduced expression of inflammatory markers. These observations were accompanied with demonstrable reductions in tissue injury and improved behavioural outcomes, indicating clear neuroprotective effects.

After stroke, the secondary overactivation of NMDARs contributes to brain damage $[12,51,52]$. Our model confirmed this finding via the increased phosphorylation of CaMKII observed after pMCAO; furthermore, the administration of S1RA decreased the NMDAR activation associated with stroke. In fact, $\sigma 1 \mathrm{R}$ forms a functional complex with the HINT1 protein at a molecular level to coordinate the activity of certain GPCRs with those of NMDARs [16]. In this scenario, the activation of these
GPCRs leads to the PKC/Src-mediated phosphorylation of NMDARs, including their subsequent separation and activation $[23,53]$. The antagonists of $\sigma 1 \mathrm{Rs}$ provoke the transfer of HINT1 proteins from activated GPCRs to NMDARs, thereby disrupting the GPCR-mediated activation of NMDARs. In our study, $\sigma 1 \mathrm{R}$ antagonists most likely prevented focal-activated GPCRs from enhancing the function of NMDARs in the surrounding area, thereby diminishing the average ischaemic damage. However, $\sigma 1 \mathrm{R}$ agonists can also reduce the effect of direct NMDAR activators. In fact, $\sigma 1 R$ agonists, in the absence of GPCR activation, can promote GPCR-NMDAR association, and this complex diminishes the activity of the associated NMDARs. Thus, $\sigma 1 \mathrm{R}$ agonists that promote the association of inactive NMDARs with silent GPCRs reduce the pool of NMDARs that are susceptible to glycine/glutamate activation.

To the best of our knowledge, our study is the first to implicate $\sigma 1 \mathrm{Rs}$ in the neuronal damage promoted by experimental stroke. We report the beneficial effects of the selective $\sigma 1 \mathrm{R}$ antagonist S1RA on stroke-induced clinically relevant gait impairments. The use of drugs to target $\sigma 1 \mathrm{R}$ is an attractive therapeutic protection strategy compared with NMDAR antagonists that have a comparatively narrow therapeutic window.

Acknowledgements This work was supported by Ministerio Economia, Industria y Competitividad, Plan Nacional I + D + I, SAF2015-65420R (JGN). We would like to thank Gabriela de Alba and Carmelo Aguado for their excellent technical assistance. We thank Alice Wareham (Aspire Scientific Ltd., Bollington, UK) for her comments on the manuscript.

\section{Compliance with Ethical Standards}

Conflict of Interest The authors declare that they have no conflict of interest.

Open Access This article is distributed under the terms of the Creative Commons Attribution 4.0 International License (http:// creativecommons.org/licenses/by/4.0/), which permits unrestricted use, distribution, and reproduction in any medium, provided you give appropriate credit to the original author(s) and the source, provide a link to the Creative Commons license, and indicate if changes were made.

\section{References}

1. Grysiewicz RA, Thomas K, Pandey DK (2008) Epidemiology of ischemic and hemorrhagic stroke: incidence, prevalence, mortality and risk factors. Neurol Clin 26:871-895. doi:10.1016/j.ncl.2008. 07.003

2. Mozaffarian D, Benjamin EJ, Go AS, Arnett DK, Blaha MJ et al (2016) Heart disease and stroke statistics - 2016 update — a report from the American Heart Association. Circulation 133:e38-360. doi:10.1161/CIR.0000000000000350

3. Appelros P, Stegmayr B, Terént A (2009) Sex differences in stroke epidemiology. A systematic review Stroke 40:1082-1090. doi:10. 1161/STROKEAHA.108.540781 
4. Dobkin BH, Dorsch A (2013) New evidence for therapies in stroke rehabilitation. Curr Atheroscler Rep 15:331. doi:10. 1007/s11883-013-0331-y

5. Cramer SC (2015) Drugs to enhance motor recovery after stroke. Stroke 46:2998-3005. doi:10.1161/STROKEAHA.115.007433

6. Brennan AM, Suh SW, Won SJ, Narasimhan P, Kauppinen TM, Lee H, Edling Y, Chan PH et al (2009) NADPH oxidase is the primary source of superoxide induced by NMDA receptor activation. Nat Neurosci 12:857-863. doi:10.1038/nn.2334

7. Gasche Y, Copin JC, Sugawara T, Fujimura M, Chan PH (2001) Matrix metalloproteinase inhibition prevents oxidative stressassociated blood-brain barrier disruption after transient focal cerebral ischemia. J Cereb Blood Flow Metab 21:1393-1400. doi:10. 1097/00004647-200112000-00003

8. Montaner J, Molina CA, Monasterio J, Abilleira S, Arenillas JF, Ribó M, Quintana M, Alvarez-Sabín J (2003) Matrix metalloproteinase-9 pretreatment level predicts intracranial hemorrhagic complications after thrombolysis in human stroke. Circulation 107:598-603 doi. Org/10.1161/01.CIR.0000046451.38849.90

9. Rosenberg GA, Estrada EY, Dencoff JE (1998) Matrix metalloproteinases and TIMPs are associated with blood-brain barrier opening after reperfusion in rat brain. Stroke 29:2189-2195 doi.Org/10. 1161/01.STR.29.10.2189

10. Takagi K, Ginsberg MD, Globus MY, Dietrich WD, Martinez E, Kraydieh S, Busto R (1993) Changes in amino acid neurotransmitters and cerebral blood flow in the ischemic penumbral region following middle cerebral artery occlusion in the rat: correlation with histopathology. J Cereb Blood Flow Metab 13:575-585. doi:10. 1038/jcbfm.1993.75

11. Benveniste H, Drejer J, Schousboe A, Diemer NH (1984) Elevation of the extracellular concentrations of glutamate and aspartate in rat hippocampus during transient cerebral ischemia monitored by intracerebral microdialysis. J Neurochem 43:1369-1374. doi:10. 1111/j.1471-4159.1984.tb05396.x

12. Lipton P (1999) Ischemic cell death in brain neurons. Physiol Rev 79:1431-1568

13. Trotman M, Vermehren P, Gibson CL, Fern R (2015) The dichotomy of memantine treatment for ischemic stroke: dose-dependent protective and detrimental effects. J Cereb Blood Flow Metab 35: 230-239. doi:10.1038/jcbfm.2014.188

14. Kourrich S, Su TP, Fujimoto M, Bonci A (2012) The sigma-1 receptor: roles in neuronal plasticity and disease. Trends Neurosci 35:762-771. doi:10.1016/j.tins.2012.09.007

15. Martina M, Turcotte ME, Halman S, Bergeron R (2007) The sigma-1 receptor modulates NMDA receptor synaptic transmission and plasticity via SK channels in rat hippocampus. J Physiol 578:143-157. doi:10.1113/jphysiol.2006.116178

16. Rodríguez-Muñoz M, Sánchez-Blázquez P, Herrero-Labrador R, Martínez-Murillo R, Merlos M, Vela JM, Garzón J (2015) The sigmal receptor engages the redox-regulated HINT1 protein to bring opioid analgesia under NMDA receptor negative control. Antioxid Redox Signal 22:799-818. doi:10.1089/ars.2014.5993

17. Vela JM, Merlos M, Almansa C (2015) Investigational sigma-1 receptor antagonists for the treatment of pain. Expert Opin Investig Drugs 24:883-896. doi:10.1517/13543784.2015.1048334

18. Kim HW, Kwon YB, Roh DH, Yoon SY, Han HJ, Kim KW, Beitz AJ, Lee JH (2006) Intrathecal treatment with sigmal receptor antagonists reduces formalin-induced phosphorylation of NMDA receptor subunit 1 and the second phase of formalin test in mice. Br J Pharmacol 148:490-498. doi:10. 1038/sj.bjp.0706764

19. Ruscher K, Shamloo M, Rickhag M, Ladunga I, Soriano L, Gisselsson L, Toresson H, Ruslim-Litrus L et al (2011) The sigma1 receptor enhances brain plasticity and functional recovery after experimental stroke. Brain 134:732-746. doi:10.1093/brain/awq367
20. Diaz JL, Cuberes R, Berrocal J, Contijoch M, Christmann U, Fernández A, Port A, Holenz J et al (2012) Synthesis and biological evaluation of the 1-arylpyrazole class of sigma(1) receptor antagonists: identification of 4-\{2-[5-methyl-1-(naphthalen-2-yl)-1Hpyrazol-3-yloxy]ethyl \}morpholine (S1RA, E-52862). J Med Chem 55:8211-8224. doi:10.1021/jm3007323

21. Carmichael ST (2005) Rodent models of focal stroke: size, mechanism, and purpose. NeuroRx 2:396-409. doi:10.1602/ neurorx.2.3.396

22. Fluri F, Schuhmann MK, Kleinschnitz C (2015) Animal models of ischemic stroke and their application in clinical research. Drug Design Dev Ther 9:3445-3454. doi:10.2147/DDDT.S56071

23. Rodríguez-Muñoz M, Cortes-Montero E, Pozo-Rodrigálvarez A, Sánchez-Blázquez P, Garzón-Niño J (2015) The ON:OFF switch, sigma1R-HINT1 protein, controls GPCR-NMDA receptor crossregulation: implications in neurological disorders. Oncotarget 6: 35458-35477. doi:10.18632/oncotarget.6064

24. Sánchez-Blázquez P, Rodríguez-Muñoz M, Herrero-Labrador R, Burgueño J, Zamanillo D, Garzón J (2014) The calcium-sensitive sigma-1 receptor prevents cannabinoids from provoking glutamate NMDA receptor hypofunction: implications in antinociception and psychotic diseases. Int J Neuropsychopharmacol 17:1943-1955. doi:10.1017/S1461145714000029

25. Winter B, Juckel G, Viktorov I, Katchanov J, Gietz A, Sohr R, Balkaya M, Hörtnagl H et al (2005) Anxious and hyperactive phenotype following brief ischemic episodes in mice. Biol Psychiatry 57:1166-1175. doi:10.1016/j.biopsych.2005.02.010

26. Hacke W, Schwab S, Horn M, Spranger M, De Georgia M, von Kummer R (1996) "Malignant" middle cerebral artery territory infarction: clinical course and prognostic signs. Arch Neurol 53:309-315. doi:10.1001/archneur.1996.00550040037012

27. Macrae IM (2011) Preclinical stroke research—advantages anddisadvantages of the most common rodent models of focal ischaemia. Br J Pharmacol 164:1062-1078. doi:10.1111/j. 1476-5381.2011.01398.x

28. Schetz JA, Perez E, Liu R, Chen S, Lee I, Simpkins JW (2007) A prototypical sigma-1 receptor antagonist protects against brain ischemia. Brain Res 1181:1-9. doi: 10.1016/j.brainres.2007.08.068

29. Su TP, London ED, Jaffe JH (1988) Steroid binding at sigma receptors suggests a link between endocrine, nervous, and immune systems. Science 240:219-221. doi:10.1126/science.2832949

30. Wali B, Ishrat T, Won S, Stein DG, Sayeed (2014) Progesterone in experimental permanent stroke: a dose-response and therapeutic time-window study. Brain 137:486-502. doi:10.1093/brain/awt319

31. Liu A, Margaill I, Zhang S, Labombarda F, Coqueran B, Delespierre B, Liere P, Marchand-Leroux C et al (2012) Progesterone receptors: a key for neuroprotection in experimental stroke. Endocrinology 153:3747-3757. doi:10.1210/ en.2012-1138

32. Gibson CL, Constantin D, Prior MJ, Bath PM, Murphy SP (2005) Progesterone suppresses the inflammatory response and nitric oxide synthase-2 expression following cerebral ischemia. Exp Neurol 193:522-530. doi:10.1016/j.expneurol.2005.01.009

33. Takahashi H, Kirsch JR, Hashimoto K, London ED, Koehler RC, Traystman RJ (1996) PPBP [4-phenyl-1-(4-phenylbutyl) piperidine] decreases brain injury after transient focal ischemia in rats. Stroke 27:2120-2123 doi.Org/10.1161/01.STR.27.11.2120

34. Takahashi H, Traystman RJ, Hashimoto K, London ED, Kirsch JR (1997) Postischemic brain injury is affected stereospecifically by pentazocine in rats. Anesth Analg 85:353-357

35. Allahtavakoli M, Jarrott B (2011) Sigma-1 receptor ligand PRE084 reduced infarct volume, neurological deficits, proinflammatory cytokines and enhanced anti-inflammatory cytokines after embolic stroke in rats. Brain Res Bull 85:219-224. doi:10. 1016/j.brainresbull.2011.03.019 
36. Shen YC, Wang YH, Chou YC, Liou KT, Yen JC, Wang WY, Liao JF (2008) Dimemorfan protects rats against ischemic stroke through activation of sigma-1 receptor-mediated mechanisms by decreasing glutamate accumulation. J Neurochem 104:558-572. doi:10.1111/j. 1471-4159.2007.05058.x

37. Ruscher K, Shamloo M, Rickhag M, Ladunga I, Soriano L, Gisselsson L, Toresson H, Ruslim-Litrus L et al (2011) The sigma-1 receptor enhances brain plasticity and functional recovery after experimental stroke. Brain 134:732-746. doi:10. 1093/brain/awq367

38. Urfer R, Moebius HJ, Skoloudik D, Santamarina E, Sato W, Mita S, Muir KW, Cutamesine Stroke Recovery Study Group (2014) Phase II trial of the sigma-1 receptor agonist cutamesine (SA4503) for recovery enhancement after acute ischemic stroke. Stroke 45: 3304-3310. doi:10.1161/STROKEAHA.114.005835

39. Vicente-Sánchez A, Sánchez-Blázquez P, Rodríguez-Muñoz M, Garzon J (2013) HINT1 protein cooperates with cannabinoid 1 receptor to negatively regulate glutamate NMDA receptor activity. Mol Brain 6:42. doi:10.1186/1756-6606-6-42

40. del Zoppo GJ, Hallenbeck JM (2000) Advances in the vascular pathophysiology of ischemic stroke. Thromb Res 98:73-81 doi: 10.1016/S0049-3848(00)00218-8

41. Turner RJ, Sharp FR (2016) Implications of MMP9 for blood brain barrier disruption and hemorrhagic transformation following ischemic stroke. Front Cell Neurosci 10:56. doi:10.3389/fncel.2016. 00056

42. Agrawal SM, Lau L, Yong VW (2008) MMPs in the central nervous system: where the good guys go bad. Semin Cell Dev Biol 19: 42-51. doi:10.1016/j.semcdb.2007.06.003

43. Asahi M, Asahi K, Jung JC, del Zoppo GJ, Fini ME, Lo EH (2000) Role for matrix metalloproteinase 9 after focal cerebral ischemia: effects of gene knockout and enzyme inhibition with BB-94. J Cereb Blood Flow Metab 20:1681-1689. doi:10.1097/00004647-200012000-00007

44. Castellanos M, Sobrino T, Millan M, García M, Arenillas J, Nombela F, Brea D, Perez de la Ossa N et al (2007) Serum cellular fibronectin and matrix metalloproteinase- 9 as screening biomarkers for the prediction of parenchymal hematoma after thrombolytic therapy in acute ischemic stroke: a multicenter confirmatory study. Stroke 38:1855-1859. doi:10.1161/STROKEAHA.106.481556

45. Montaner J, Alvarez-Sabin J, Molina CA, Anglés A, Abilleira S, Arenillas J, Monasterio J (2001) Matrix metalloproteinase expression is related to hemorrhagic transformation after cardioembolic stroke. Stroke 32:2762-2767. doi.Org/10.1161/hs1201.99512

46. Kim JY, Kawabori M, Yenari MA (2008) Innate inflammatory responses in stroke: mechanisms and potential therapeutic targets. Curr Med Chem 21:2076-2097. doi:10.2174/ 0929867321666131228205146

47. Lai AY, Todd KG (2006) Microglia in cerebral ischemia: molecular actions and interactions. Can J Physiol Pharmacol 84:49-59. doi: $10.1139 / Y 05-143$

48. Sofroniew MV (2009) Molecular dissection of reactive astrogliosis and glial scar formation. Trends Neurosci 32:638-647. doi:10. 1016/j.tins.2009.08.002

49. Yang Z, Wang KK (2015) Glial fibrillary acidic protein: from intermediate filament assembly and gliosis to neurobiomarker. Trends in Neuroscience 38:364-374. doi:10.1016/j.tins.2015.04.003

50. Burda JE, Sofroniew MV (2014) Reactive gliosis and the multicellular response to CNS damage and disease. Neuron 81:229-248. doi:10.1016/j.neuron.2013.12.034

51. Lee JM, Zipfel GJ, Choi DW (1999) The changing landscape of ischaemic brain injury mechanisms. Nature 399:A7-14. doi:10. 1038/399a007

52. Moskowitz MA, Lo EH, Iadecola C (2010) The science of stroke: mechanisms in search of treatments. Neuron 67:181-198. doi:10. 1016/j.neuron.2010.07.002

53. Rodríguez-Muñoz M, Sánchez-Blázquez P, Merlos M, Gazon-Niño J (2016) Endocannabinoid control of glutamate NMDA receptors: the therapeutic potential and consequences of dysfunction. Oncotarget 7:55840-55862. doi:10.18632/oncotarget.10095 